\title{
Pentamode-Based Coding Metasurface for Underwater Acoustic Stealth
}

\author{
Li He, Li Cai, Xing Chen \\ Science and Technology on Integrated Logistics Support Laboratory, National University of Defense Technology, Changsha, China \\ Email: 245181437@qq.com
}

How to cite this paper: He, L., Cai, L. and Chen, X. (2021) Pentamode-Based Coding Metasurface for Underwater Acoustic Stealth. Journal of Applied Mathematics and Physics, 9, 1829-1836.

https://doi.org/10.4236/jamp.2021.97117

Received: July 6, 2021

Accepted: July 27, 2021

Published: July 30, 2021

\begin{abstract}
Coding metasurface draws amounts of research interests due to its potential for achieving sophisticated functions in wave manipulation by using simple logical unit cells with out-of-phase responses. In this paper, we present a novel acoustic coding metasurface structure for underwater sound scattering reduction based on pentamode metamaterials. The metasurface is composed of two types of hexagonal pentamode unit cells with phase responses of 0 and $\pi$ respectively. The units are arranged in random 1-bit coding sequence to achieve low-scattering underwater acoustic stealth effect. Full-wave simulation results are in good accordance with the theoretical expectation. The optimized arrangement resulted in the distribution of scattered underwater acoustic waves and suppression of the far field scattering coefficient over a wide range of incident angles. We show that pentamode-based coding metasurface provides an efficient scheme to achieve underwater acoustic stealth by ultrathin structures.
\end{abstract}

\section{Keywords}

Pentamode Metamaterials, Coding Metasurface Structure, Underwater Sound Scattering

\section{Introduction}

It is a significant topic to manipulate the propagation of sound at will. Such a design is available by employing artificial acoustic metamaterials. Recently, as the planarized counterparts of the acoustic metamaterials, acoustic metasurfaces, inspired from the pioneering works on optical metasurfaces, are attracting extensive attention due to their capabilities in controlling sound wavefronts [1]-[6]. Through assembling arrays of deep subwavelength units with carefully designed gradient phase profile, numerous fascinating phenomena have been 
observed, including anomalous refraction/reflection, beam bending, converting propagating waves to surface waves, acoustic diode, and vortex beam [7]-[12]. Due to its reduced dimensionality and possibility to effectively control waves, the metasurface may become a good candidate to design acoustic components.

Recently, a new concept of manipulation waves by artificial subwavelength structures, termed the digital or coding metasurfaces, has been developed for the flexibility in the electromagnetic and acoustic wave control [13]. It contains only limited kinds of building elements (typically structures contain only two kinds of structure units to give rise to the phases of " 0 " and " $\pi$."), called Boolean numbers or logical bits, to realize different complicated functions by assembling the elements in a binary like way [14] [15] [16]. It can be viewed as a bridge linking between metasurface and digital perspective and has more freedoms than the traditional metasurfaces. Moreover, by introducing reconfigurable unit cells, the coding can be controlled to realize real-time control of electromagnetic waves or acoustic waves. Several interesting studies have been reported [17] [18] [19] [20] [21], including RCS control, reverberating scenarios, computational imaging and wavefront and scattering signature.

In this paper, we aim to a pentamode-based underwater acoustic coding metasurface design. A digital metasurface model composed of different pentamode units is proposed to realize underwater acoustic stealth based on the destructive interferences. The units are arranged in random 1-bit coding sequence and the scattered field due to an incident wave would be distributed uniformly among all possible directions. A kind of two-dimensional (2D) hexagonal pentamode metamaterial is introduced to obtain the required phase response [22] [23]. The distribution of scattered sound field under plane wave incidence are studied and it has been found that the optimized arrangement resulted in a considerable reduction in the far field scattering coefficient over a wide angle range. We show that pentamode-based coding metasurface may be a novel way to achieve underwater acoustic stealth by ultrathin structures.

\section{Model and Theory}

Figure 1 illustrates the basic idea and geometry of the coding metasurfaces under investigation. We assume a planar structure in the $x-y$ plane and a plane acoustic wave incident into it from top to bottom, just as shown in Figure 1. The planar structure is backed by a steel backing and with water (speed of sound $c_{0}=$ $1490 \mathrm{~m} / \mathrm{s}$; densit $\mathrm{y}_{0}=1000 \mathrm{~kg} / \mathrm{m}^{3}$ ) as the background medium. The planar metasurface consists of periodically repeated rectangle cells. The thickness and period of the cells are $d$ and $L$, respectively. The ideal cells are filled with impedance-matched inhomogeneous fluidlike materials and can be featured by two possible element types with different velocity or index. When acoustic waves are reflected from the two cells, they should be with the same amplitudes but the phase differences between the different elements should be $\pi$ at the working bandwidth, corresponding to the " 0 " and "1" elements respectively. 


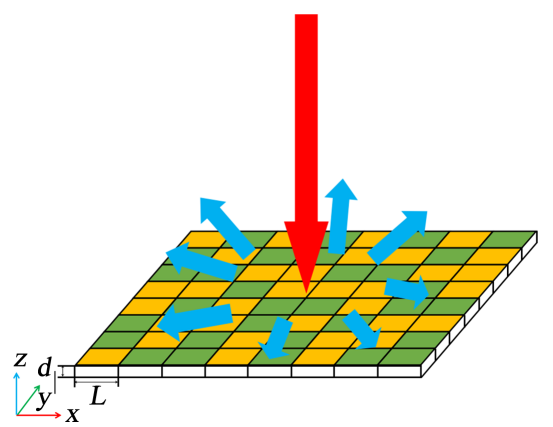

Figure 1. The schematic of the planar coding metasurface composed of squared coding element " 0 " and " 1 ". Arrows indicate the incident and reflected waves.

Pentamode material (PM) is a kind of special metamaterial with five of the six eigenvalues of its modulus matrix are nearly zero. This means that it is much easier to change the shape of such a metamaterial (while fixing its volume) than it is to change its volume (while fixing its shape) and has similar mechanical properties to those of water, with a very high bulk modulus but a very low shear modulus and is also called "metafluid" or metal water. More important, its mechanical properties are much more dependent on the designed structure than on the chemical composition, so the effective bulk modulus and mass density can be adjusted conveniently by vary its structure parameters of the sub-wavelength unit cells. It makes the pentamode materials is especially suitable for controlling underwater acoustics. Here two kinds of two-dimensional (2D) hexagonal pentamode unit cells are introduced to build the proposed metasurface, just as shown in Figure 2, which are network of metallic arms arranged in a regular honeycomb lattice (with a thickness of $t$ and a side length $l_{1}$ ), with six additional weights (with a width of $l$, a height of $h$ ) located at the vertices of the hexagon. The " 1 " and "0" element-types are implemented via suitable choices of the width of the interconnecting arms, the length and the width of the star arms patch sidelength. The effective mass density $\rho_{\text {eff }}$ approximates its bulk average density, while its effective bulk modulus $k_{\text {eff }}$ can be calculated by Gibson formula [24] as follow:

$$
\begin{aligned}
& k_{\text {eff }}=E_{\text {solid }} \frac{4}{\sqrt{3}}\left(\frac{t}{l_{1}}\right)^{3} \\
& \rho_{\text {eff }}=2 \frac{\rho_{\text {solid }}\left(3 \sqrt{3}\left(l_{1}-t / \sqrt{3}\right)^{2} / 2\right)+\rho_{\text {solid }} 6 h l}{3 \sqrt{3} l_{1}^{2}}
\end{aligned}
$$

where the unit cells are made of steel with density $\rho_{\text {steel }}=7800 \mathrm{~kg} / \mathrm{m}^{3}$ and modulus $E_{\text {steel }}=210 \mathrm{GPa}$. These relations imply the acoustic metasurfaces with the spatially varying parameter profile can be realized by tailoring the geometry of the pentamode units. A total of 50 sectors of cells are assembled and we consider the same unitcell geometry ( $h$ and $l$ are the same), the " 1 " and " 0 " element-types are implemented via suitable choices of $t, h_{1}$ and $l_{1}$ to obtain required effective velocities, as well as the corresponding effective mass densities is implement to keep impendance matching with the surrounding medium. 

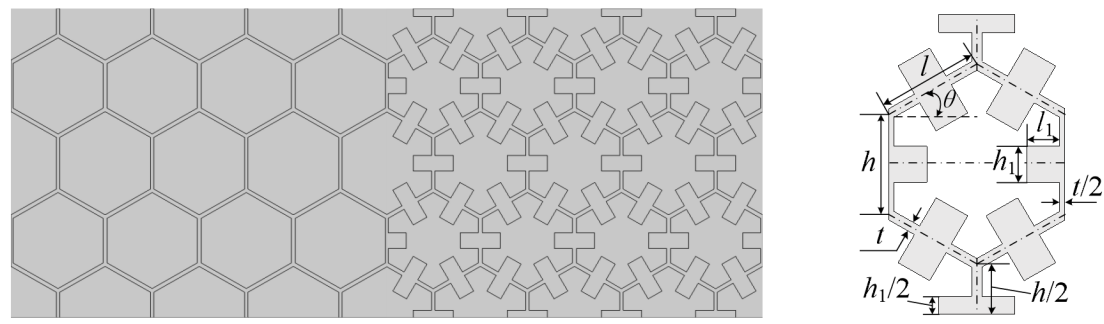

Figure 2. Schematic diagram of the metasurface composed of pentamode units, the two structures are " 0 " unit and " 1 " unit respectively. Another one is schematic diagram of the pentamode unit.

\section{Results and Discussion}

Reducing the scattering of waves is widely concerned in both electromagnetic waves and acoustic stealth field. Several methods, such as wave absorbing materials, scattering cansellation, and cloak have been widely used or studied. In the field of coding metasurfaces, a new approach proposed to reducing the scattering of waves is to generate a phase-distribution matrix with "0" and " 1 " elements randomly distributed, and place each element according to its reflection phase, just as shown in Figure 1. The two coding elements are shown in the Figure 2. Under the normal incidence of the plane waves, the far-field distribution of the waves scattered by the metasurface can be expressed as

$$
\begin{aligned}
& f(\theta, \varphi)=f_{e}(\theta, \varphi) \\
& \sum_{m=1}^{N} \sum_{n=1}^{N} \exp \left\{-i\left\{\varphi(m, n)+k L \sin \theta\left[\left(m-\frac{1}{2}\right) \cos \varphi+\left(n-\frac{1}{2}\right) \sin \varphi\right]\right\}\right\}
\end{aligned}
$$

where $k$ is the wave vector, $\theta$ and $\varphi$ are the elevation and azimuth angles of an arbitrary direction, respectively, and $f_{e}(\theta, \varphi)$ is the pattern function of the array.

Here a 1-bit coding metasurface composed of pentamode coding elements is designed with random coding sequence. Firstly, an $16 \times 16$ array of coding sequence is randomly generated by MATLAB as shown in the following:

$$
\left[\begin{array}{llllllllllllllll}
1 & 0 & 1 & 0 & 0 & 0 & 1 & 0 & 1 & 0 & 0 & 0 & 0 & 1 & 0 & 1 \\
0 & 0 & 0 & 0 & 0 & 0 & 0 & 1 & 1 & 0 & 1 & 1 & 0 & 0 & 1 & 0 \\
0 & 0 & 1 & 0 & 0 & 1 & 1 & 1 & 0 & 0 & 1 & 0 & 1 & 1 & 0 & 0 \\
1 & 1 & 0 & 1 & 1 & 0 & 0 & 0 & 1 & 1 & 0 & 1 & 0 & 1 & 0 & 1 \\
1 & 0 & 0 & 1 & 0 & 0 & 1 & 1 & 0 & 0 & 1 & 1 & 1 & 0 & 0 & 1 \\
0 & 0 & 1 & 1 & 1 & 0 & 1 & 1 & 0 & 0 & 0 & 1 & 1 & 1 & 1 & 0 \\
1 & 1 & 0 & 0 & 1 & 0 & 1 & 0 & 1 & 0 & 0 & 0 & 1 & 0 & 0 & 1 \\
0 & 0 & 1 & 0 & 1 & 1 & 1 & 0 & 0 & 1 & 0 & 1 & 0 & 0 & 0 & 1 \\
1 & 1 & 1 & 0 & 0 & 0 & 0 & 1 & 0 & 1 & 1 & 1 & 1 & 0 & 1 & 1 \\
1 & 0 & 0 & 1 & 1 & 0 & 0 & 0 & 1 & 0 & 0 & 1 & 1 & 1 & 0 & 1 \\
0 & 0 & 0 & 0 & 1 & 0 & 0 & 0 & 1 & 1 & 1 & 1 & 0 & 0 & 0 & 0 \\
0 & 0 & 1 & 0 & 1 & 1 & 1 & 0 & 1 & 0 & 1 & 0 & 1 & 1 & 0 & 1 \\
0 & 0 & 1 & 0 & 1 & 1 & 0 & 0 & 0 & 1 & 0 & 1 & 1 & 1 & 1 & 1 \\
0 & 1 & 1 & 1 & 1 & 0 & 0 & 0 & 1 & 1 & 0 & 1 & 0 & 1 & 1 & 1 \\
1 & 0 & 1 & 1 & 1 & 1 & 1 & 0 & 1 & 0 & 1 & 0 & 1 & 0 & 1 & 1 \\
0 & 0 & 1 & 1 & 0 & 1 & 0 & 0 & 1 & 1 & 1 & 0 & 1 & 1 & 0 & 0
\end{array}\right]
$$


Then, according to the random coding sequence, a metasurface is constructed with pentamode unit cells. Using COMSOL software simulation, we get the far-field scattering pressure field, as shown in Figure 3.

From Figure 3, we can see that the 1-bit random coding metasurface composed of pentamode coding elements can effectively disperse the scattering of sound waves and make the reflected sound waves scatter in different directions, so as to reduce the scattering intensity of sound waves. For further research, we calculated the far-field scattering pressure field of the flat plate and the 1-bit random coding metasurface composed of pentamode coding elements. The results are shown in Figure 4. Through the results, we can clearly see that the
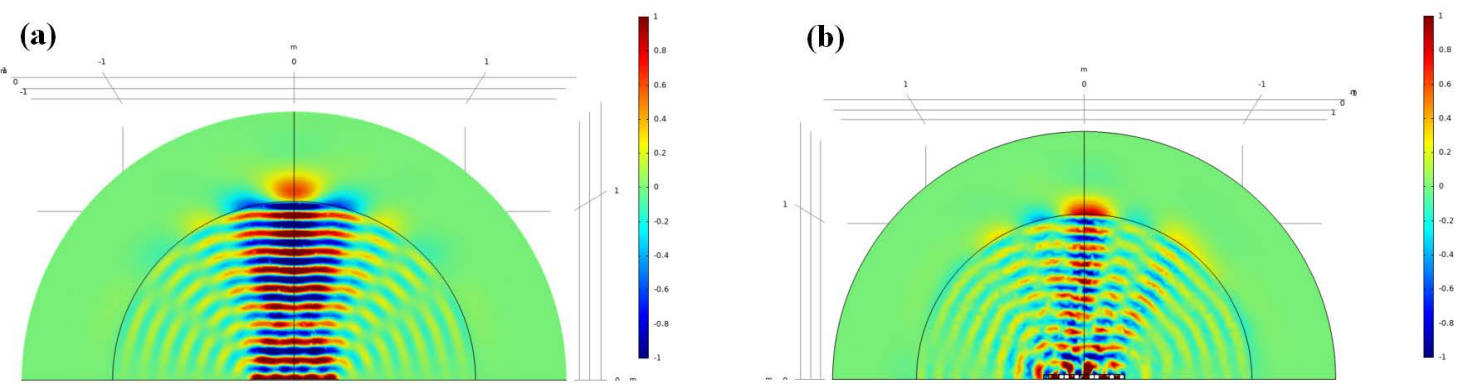

Figure 3. The simulated underwater 3D far-field scattering pattern at $7450 \mathrm{~Hz}$. (a) Underwater acoustic scattering from a flat plate. (b) Underwater acoustic scattering from 1-bit coding metasurface composed of pentamode coding elements is designed with random coding sequence.
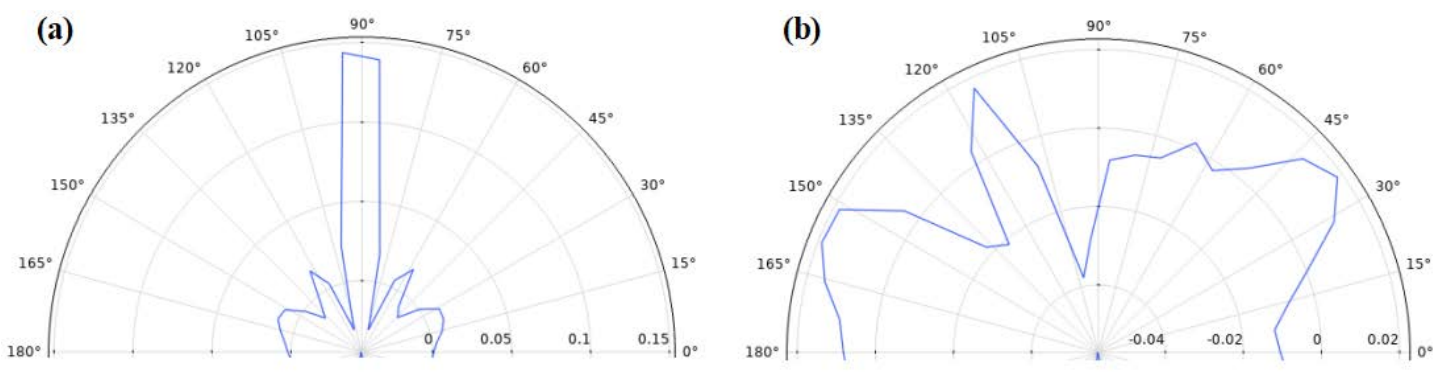

(c)

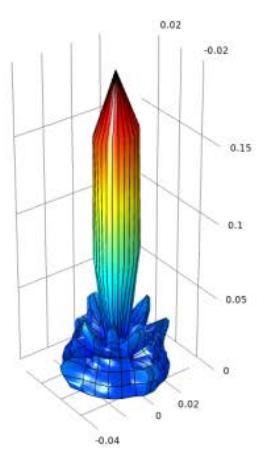

(d)

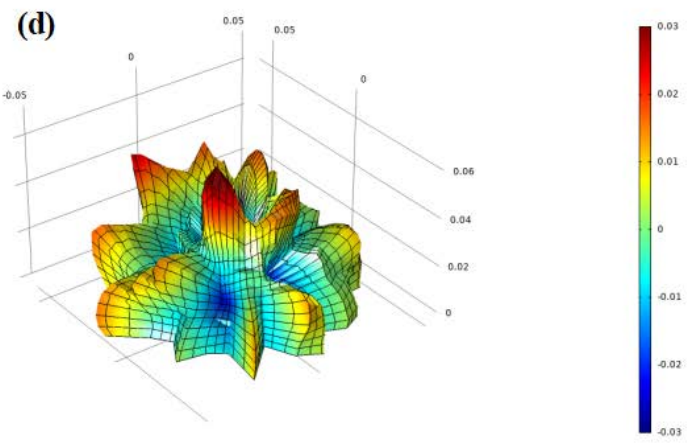

Figure 4. The simulated underwater far-field scattering pressure pattern of sound waves at $7450 \mathrm{~Hz}$. (a) Underwater 2D far-field scattering pressure pattern of sound waves from a flat plate. (b) Underwater 2D far-field scattering pressure pattern of sound waves form 1-bit coding metasurface composed of pentamode coding elements is designed with random coding sequence. (c) Underwater 3D far-field scattering pressure pattern of sound waves form a flat plate. (b) Underwater 3D far-field scattering pressure pattern of sound waves form 1-bit coding metasurface composed of pentamode coding elements is designed with random coding sequence. 

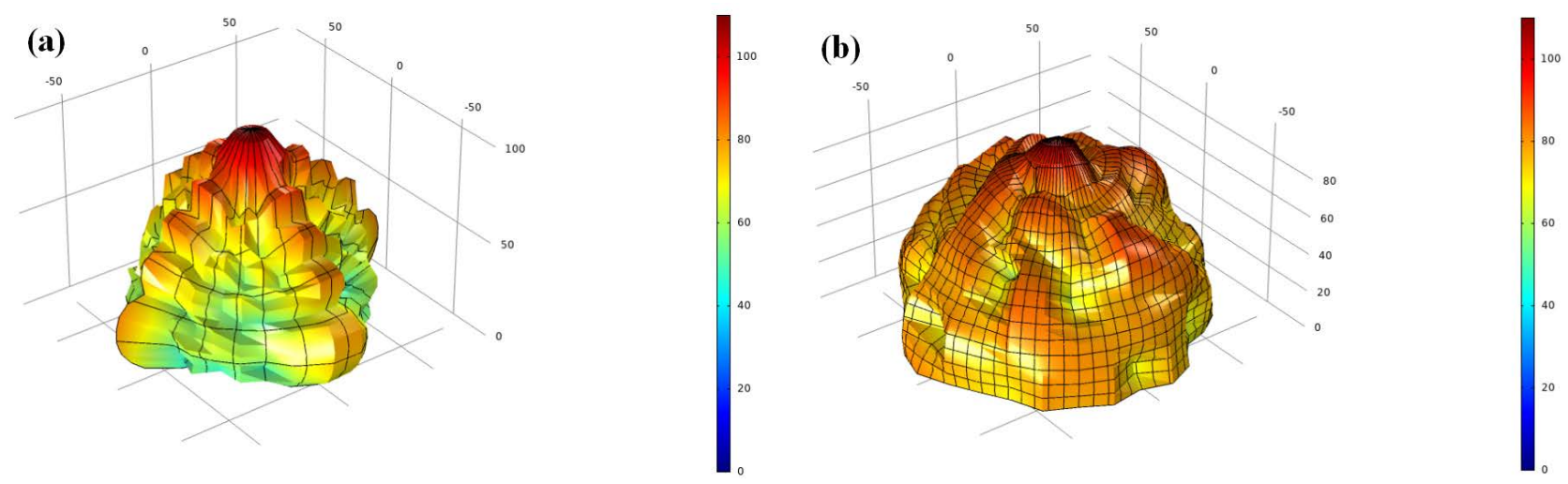

Figure 5. The simulated underwater far-field scattering pressure level of sound waves at $7450 \mathrm{~Hz}$. (a) Underwater 3D far-field scattering pressure level of sound waves from a flat plate. (b) Underwater 3D far-field scattering pressure level of sound waves form 1-bit coding metasurface composed of pentamode coding elements is designed with random coding sequence.

far-field scattering pressure field of the 1-bit random coding metasurface composed of pentamode coding elements is dispersed. Compared with the flat plate, it greatly reduces the far-field scattering pressure.

Finally, we simulate the underwater far-field scattering pressure level of sound waves on the 1-bit random coding metasurface composed of pentamode coding elements, and the results are shown in Figure 5. Through simulation, we get that the far-field scattering sound pressure level of the 1-bit random coding metasurface composed of pentamode coding elements is reduced by about $10 \mathrm{~dB}$ compared with that of the flat plate.

\section{Conclusion}

In conclusion, a low-scattering underwater acoustic coding metasurface is proposed based on pentamode metamaterials. The metasurface is composed of two types of hexagonal pentamode unit cells with phase responses of 0 and $\pi$ respectively. The units are arranged in random 1-bit coding sequence to make the scattered field due to an incident wave would be distributed uniformly in all possible directions. Studies have shown that the optimized arrangement of the random coding sequence can result in a considerable reduction in the far field scattering coefficient over a wide angle range. Our research works provide an efficient scheme for the application of coding acoustic metasurfaces in underwater sound manipulation, which may promise potential applications in underwater acoustic stealth, focusing, and detecting.

\section{Conflicts of Interest}

The authors declare no conflicts of interest regarding the publication of this paper.

\section{References}

[1] Li, Y., Liang, B., Gu, Z.M., Zou, X.Y. and Cheng, J.C. (2013) Reflected Wavefront Manipulation Based on Ultrathin Planar Acoustic Metasurfaces. Scientific Reports, 


\section{3, 2546. https://doi.org/10.1038/srep02546}

[2] Zhao, J.J., Li, B.W., Chen, Z.N. and Qiu, C.W. (2013) Redirection of Sound Waves Using Acoustic Metasurface. Applied Physics Letters, 103, 151604.

https://doi.org/10.1063/1.4824758

[3] Zhai, S., Chen, H., Ding, C., Shen, F., Luo, C. and Zhao, X. (2015) Manipulation of Transmitted Wave Front Using Ultrathin Planar Acoustic Metasurfaces. Applied Physics A, 120, 1283-1289. https://doi.org/10.1007/s00339-015-9379-6

[4] Mei, J. and Wu, Y. (2014) Controllable Transmission and Total Reflection through an Impedance-Matched Acoustic Metasurface. New Journal of Physics, 16, 123007. https://doi.org/10.1088/1367-2630/16/12/123007

[5] Zhao, J.J., Li, B.W., Chen, Z.N. and Qiu, C.W. (2013) Redirection of Sound Waves Using Acoustic Metasurface. Applied Physics Letters, 103, 151604. https://doi.org/10.1063/1.4824758

[6] Zuo, S.Y., Tian, Y., Wei, Q., Cheng, Y. and Liu, X.J. (2018) Acoustic Analog Computing Based on a Reflective Metasurface with Decoupled Modulation of Phase and Amplitude. J. Appl. Phys, 123, 091704. https://doi.org/10.1063/1.5004617

[7] Yu, N., Genevet, P., Kats, M.A., Aieta, F., Tetienne, J.-P., Capasso, F. and Gaburro, Z. (2011) Light Propagation with Phase Discontinuities: Generalized Laws of Reflection and Refraction. Science, 334, 333. https://doi.org/10.1126/science.1210713

[8] Xie, Y., Wang, W., Chen, H., Konneker, A., Popa, B.-I. and Cummer, S.A. (2014) Wavefront Modulation and Subwavelength Diffractive Acoustics with an Acoustic Metasurface. Nature Communications, 5, 5553. https://doi.org/10.1038/ncomms6553

[9] Tang, K., Qiu, C., Ke, M., Lu, J., Ye, Y. and Liu, Z. (2014) Anomalous Refraction of Airborne Sound through Ultrathin Metasurfaces. Sci. Rep., 4, 6517.

https://doi.org/10.1038/srep06517

[10] Ding, C., Chen, H., Zhai, S., Liu, S. and Zhao, X. (2015) The Anomalous Manipulation of Acoustic Waves Based on Planar Metasurface with Split Hollow Sphere. J. Phys. D: Appl. Phys., 48, 045303. https://doi.org/10.1088/0022-3727/48/4/045303

[11] Li, Y., Jiang, X., Li, R., Liang, B., Zou, X., Yin, L. and Cheng, J. (2014) Experimental Realization of Full Control of Reflected Waves with Subwavelength Acoustic Metasurfaces. Phys. Rev. Appl., 2, 064002. https://doi.org/10.1103/PhysRevApplied.2.064002

[12] Tang, K., Qiu, C., Lu, J., Ke, M. and Liu, Z. (2015) Focusing and Directional Beaming Effects of Airborne Sound through a Planar Lens with Zigzag Slits. J. Appl. Phys., 117, 024503. https://doi.org/10.1063/1.4905910

[13] Cui, T.J., Qi, M.Q., Wan, X., Zhao, J. and Cheng, Q. (2014) Coding Metamaterials, Digital Metamaterials and Programmable Metamaterials. Light. Sci. Appl., 3, e218. https://doi.org/10.1038/1sa.2014.99

[14] Giovampaola, C.D. and Engheta, N. (2014) Digital Metamaterials. Nat. Mater., 13, 1115. https://doi.org/10.1038/nmat4082

[15] Paquay, M., Iriarte, J., Ederra, I., Gonzalo, R. and de Maagt, P. (2007) Thin AMC Structure for Radar Cross-Section Reduction. IEEE Transactions on Antennas and Propagation, 55, 3630-3638. https://doi.org/10.1109/TAP.2007.910306

[16] Kaina, N., Dupré, M., Lerosey, G. and Fink, M. (2014) Shaping Complex Microwave Fields in Reverberating Media with Binary Tunable Metasurfaces. Sci Rep, 4, 6693. https://doi.org/10.1038/srep06693

[17] Li, Y.B., Li, L.L., Xu, B.B., Wu, W., Wu, R.Y., Wan, X., Cheng, Q. and Cui, T.J. 
(2016) Transmission-Type 2-Bit Programmable Metasurface for Single-Sensor and Single-Frequency Microwave Imaging. Sci Rep, 6, 23731. https://doi.org/10.1038/srep23731

[18] Zhang, X.G., Jiang, W.X., Tian, H.W. and Cui, T.J. (2018) Controlling Radiation Beams by Low-Profile Planar Antenna Arrays with Coding Elements. ACS Omega, 3, 10601-10611. https://doi.org/10.1021/acsomega.8b01679

[19] Chang, L., Li, Y., Zhang, Z. and Feng, Z. (2018) Reconfigurable 2-Bit Fixed-Frequency Beam Steering Array Based on Microstrip Line. IEEE Trans. Antennas Propag., 66, 683-691. https://doi.org/10.1109/TAP.2017.2776960

[20] Gao, L.-H., Cheng, Q., Yang, J., Ma, S.-J., Zhao, J., Liu, S., Chen, H.-B., He, Q., Jiang, W.-X., Ma, H.-F., Wen, Q.-Y., Liang, L.-J., Jin, B.-B., Liu, W.-W., Zhou, L., Yao, J.-Q., Wu, P.-H. and Cui, T.-J. (2015) Broadband Diffusion of Terahertz Waves by Multi-Bit Coding Metasurfaces. Light Sci Appl, 4, e324. https://doi.org/10.1038/1sa.2015.97

[21] Liang, L., Qi, M., Yang, J., Shen, X., Zhai, J., Xu, W., Jin, B., Liu, W., Feng, Y., Zhang, C., Lu, H., Chen, H.-T., Kang, L., Xu, W., Chen, J., Cui, T.J., Wu, P. and Liu, S. (2015) Anomalous Terahertz Reflection and Scattering by Flexible and Conformal Coding Metamaterials. Adv. Opt. Mater., 3, 1374.

https://doi.org/10.1002/adom.201500206

[22] Tian, Y., Wei, Q., Cheng, Y., Xu, Z. and Liu, X.J. (2015) Broadband Manipulation of Acoustic Wavefronts by Pentamode Metasurface. Applied Physics Letters, 107, 221906. https://doi.org/10.1063/1.4936762

[23] Lu, Z.M., Cai, L., Wen, J.-H. and Chen, X. (2019) Physically Realizable Broadband Acoustic Metamaterials with Anisotropic Density. CHIN. PHYS. LETT., 36, 024301. https://doi.org/10.1088/0256-307X/36/2/024301

[24] Fu, M.H. and Yin, J.R. (1999) Equivalent Elastic Parameters of the Honeycomb Core. Acta Mechanica Sinica, 31, 113-118. (In Chinese) 